\title{
A novel variant of the FOXC1 gene causes Axenfeld-Rieger syndrome with congenital glaucoma in a Chinese boy
}

\author{
Min Tang ${ }^{1}$, Kaiming $\mathrm{Li}^{1}$, Qi Zhou ${ }^{1}$, Jinchuan $\mathrm{Wu}^{1}$, Yang $\mathrm{Cao}^{1}$, Xiaohong Xiang ${ }^{1}$, Yingqing \\ Lei $^{1}$, Min Tian ${ }^{1}$, Lu Guo ${ }^{1}$, Yinggui $\mathrm{Yu}^{1}$, and Hongbin $\mathrm{LV}^{1}$ \\ ${ }^{1}$ The Affiliated Hospital of Southwest Medical University
}

June 3, 2020

\begin{abstract}
Axenfeld-Rieger syndrome is an autosomal dominant genetic disease characterized by binocular anterior segment development defects and systemic dysplasia. In our study, a Chinese Axenfeld-Rieger syndrome pedigree was analyzed. After obtaining consent from the subjects, ophthalmic examinations were performed, and blood samples were collected. Then, the causative gene was identified by targeted next-generation sequencing, and candidate mutations were verified by Sanger sequencing. Pathogenicity analysis and conservative analysis were performed on the mutant gene to evaluate its pathogenicity, and SWISS-MODEL was used to construct the three-dimensional structure of the FOXC1 region to predict the effect of mutations on the structure of the FOXC1 protein. Optimistically, a novel heterozygous, deleterious variant of the FOXC1 gene, c.246C >A(p.S82R), was successfully identified in this Axenfeld-Rieger syndrome pedigree, which cosegregated with the clinical phenotype. This variant resulted in the mutation of amino acid 82 from serine to arginine. The evolution of serine(s) in different species was highly conserved. This mutation led to a change in the three-dimensional structure of the protein, which was pathogenic. The discovery of these new mutation sites further expands its mutation spectrum. Our understanding of Axenfeld-Rieger syndrome will facilitate the development of methods for the diagnosis, prevention and genetic counseling of this disease.
\end{abstract}

\section{BACKGROUND}

Axenfeld-Rieger syndrome (ARS) is a rare autosomal dominant genetic disease with an incidence of 1:20000. Its main clinical symptoms are the developmental malformation of the binocular anterior segment, which is associated with craniofacial, umbilical and dental abnormalities (Sauer et al.,2012; Ito et al.,2014). There are three common clinical variants of this disease; defects that are limited to the peripheral part of the anterior eye segment are called Axenfeld anomalies. Abnormal iris hypoplasia combined with abnormalities in the periphery of the anterior eye segment is referred to as Rieger anomaly, and combined ocular abnormalities and systemic developmental defects are referred to as Axenfeld-Rieger syndrome.

ARS is often considered autosomal dominant; some sporadic cases have also been reported. ARS has genetic heterogeneity, and two pathogenic genes of ARS, FOXC1 and PITX2, are located in the chromosome 6p25 and 4q25 regions, respectively (Seifi et al.,2018; Seifi al.,2018b). Four families with diseases of the anterior eye segment (including ARS) were reported, all of which were associated with mutations in the FOXC1 gene located on chromosome 6p25. Through sequencing analysis, Nishimura et al. found three missense mutations and a small fragment deletion of $11 \mathrm{bp}$ in the FOXC1 gene.

The FOXC1 gene is located in the region of chromosome 6p25, and the protein product of FOXC1 from the $\mathrm{N}$ end to the $\mathrm{C}$ end is the active domain 1 (AD1), forkhead domain (FHD) and active domain 2 (AD2) (Berryet al.,2007). The FHD is the DNA binding region for the FOXC1 protein. Missense mutations in FHD significantly reduce protein stability and transactivation capability and the ability of FOXC1 to bind to DNA. 
Transcriptional activity and protein expression of FOXC1 are largely regulated by posttranscriptional levels (Saleem et al.,2004).

Because of the heterogeneity of the ARS phenotype and genotype and the overlap with the clinical manifestation of anterior segment dysgenesis (ASD), challenges exist for accurate diagnosis and classification (Deml et al.,2014). A correct diagnosis is often established only when the mutation in one of the known associated genes is identified. In the Chinese population, FOXC1 mutations in patients with ARS have not been well described. Therefore, the purpose of this study was to identify a new heterozygous mutation of the FOXC1 gene with ARS by using targeted next-generation sequencing technology combined with bioinformatics and expression profiling.

\section{METHOD}

\subsection{Ethical approval}

This study was approved by the Ethics Committee of Southwest University. Written informed consent was obtained from the participants or their guardians in accordance with the guidelines of the Declaration of Helsinki.

\subsection{Pedigree, proband and clinical assessment}

The pedigree consisted of a proband (Fig. 1), the proband's normal father, normal mother, normal brother and normal grandmother in a Chinese family from Luzhou, a city in Sichuan Province. For clinical assessment, a clinical history and ophthalmic examination of the proband were performed, including best-corrected visual acuity, intraocular pressure, slit lamp biological microscopy, ultrawide angle fundus imaging, optical coherence tomography, and UBM, as well as systemic examinations including heart ultrasound.

\subsection{Blood sampling and DNA extraction}

In this study, $2 \mathrm{~mL}$ of fresh peripheral blood was collected from the proband and pedigree members, and peripheral venous blood genomic DNA (gDNA) was extracted using the Wizard Genomic DNA Extraction Kit(Promega). DNA was measured using a Nanodrop 2000, and then the next experiment was carried out after quality testing criteria were met. In addition, blood samples were also taken from 100 ethnically matched and healthy control volunteers without any disease history.

\subsection{Capture panel design and target sequencing}

The main procedure of target region capture sequencing was using the capture chip to enrich the DNA fragments in the target region and then sequencing them with the help of a high-throughput next-generation sequencing platform. In our study, 50 known candidate genes for iris disease were captured by MyGenostics (GenCap). The process of capture was as follows: genomic DNA was randomly broken into fragments and linked to the Illumina PE junction oligonucleotide mixture. The products were amplified and purified by ligation-mediated polymerase chain reaction (LM-PCR), and then the DNA libraries were obtained and inspected. The above PCR product was hybridized with the target region capture chip to enrich the target region sequence. The captured sequences were sequenced on the Illumina HiSeq 2000 sequencing platform (Illumina, San Diego, CA, USA), and the original data were initially processed, including image recognition and sample division.

\subsection{PCR amplifications and Sanger sequencing}

The selected mutation sites were identified by PCR and Sanger sequencing. Primers were designed using the online software Primer 3.0. (http://primer3.ut.ee/), and the primer sequences were as follows: upstream primer, 5' GGGAGTGGTGCCCTACCT 3' downstream primer, 5’ ACTGGTAGATGCCGTTCAGG 3' (Table 3). The PCR amplification reaction system was configured using the preset program of the Biomek $(\mathbb{B}$ automatic workstation. After sufficient mixing and centrifugation, the sample was placed into a preprogrammed PCR instrument, and the program was run according to the following steps: $96^{\circ} \mathrm{C}$ for 1 min $30 \mathrm{~s} ; 96^{\circ} \mathrm{C}$ for $15 \mathrm{~s}, 50^{\circ} \mathrm{C}$ for $6 \mathrm{~s}$, and $60^{\circ} \mathrm{C}$ for $3 \mathrm{~min} 30 \mathrm{~s}$ for $28 \mathrm{cycles}$; followed by a $4^{\circ} \mathrm{C}$ hold. The PCR products were 
sequenced using Sanger sequencing and analyzed on the ABI 3130 Genetic Analyzer (Applied Biosystems), and then cosegregation analysis was performed among family members.

2.6 Protein structure and bioinformatics analysis

The original sequencing data were stripped of contamination and linker sequences and then BWA software(http://bio-bwa.sourceforge.net/) was used to compare the filtered sequences to Human Genome Reference by NCBI (hg19). GATK software (https://software.broadinstitute.org/gatk/) was used to analyze the information of single nucleotide variations (SNVs) and inserts and deletions(INDEL). All SNPs and INDELs were then annotated using ANNOVAR software (http://annovar.openbioinformatics. org/en/latest/). Mutation sites with frequencies less than 0.02 were screened out from the normal population database, which included the 1000 Genomes Project (http://www.1000genomes.org/), Exome Variant Server () and EXAC (http://exac. broadinstitute.org/).Software SIFT (http://sift.jcvi.org/), PolyPhen-2 (http://genetics.bwh.Harvard.edu/pph2/ ), MutationTaster (/) and GERP++ (http://mendel.stanford.edu/SidowLab/ downloads/gerp/index.html) software were used to predict the pathogenicity and conservatism of missense mutations, and the pathogenicity of splice site changes was analyzed using SPIDEX (http://www.deepgenomics.com/spidex) software.

2.7 Predictive analysis of the 3D model of the protein

The prediction software SWISS-MODEL (https://swissmodel.expasy.

org/interactive) was used for prediction analysis of the 3D model of the protein. The steps were as follows: input the protein sequence to be predicted and click the built model; select the model: selection covered the range of target sites; the identity and similarity of the model were not less than $30 \%$ (recommended value). The predicted model was applied using Swiss-PdbViewer (http://www.genebee.msu.su/spdbv/text/getpc.htm), a visual analysis software, and the results were obtained.

\section{RESULITS}

\subsection{Pedigree and clinical characteristics}

The proband included in our study (Fig. 1) was a 7-year-old boy from a Chinese family who was born at full term by spontaneous labor, and his binocular eyeball was large at birth. He was admitted to the hospital due to a further reduction in his visual acuity at 2 years of age and diagnosed with "congenital glaucoma", and trabeculectomy for glaucoma in both eyes was performed. His best-corrected vision was 20/200 in the right eye and 20/133 in the left eye. IOP measured with the Goldmann tonometer was $34 \mathrm{mmHg}$ in the right eye and $20 \mathrm{mmHg}$ in the left eye. The operation history included ventricular septal defect repair in our hospital more than 4 years ago and sublingual cystectomy in our hospital 2 years ago. Figure 2 shows two photographs of the anterior segment of the proband's eyes. The sclera of both eyes was pale blue, the cornea diameter was approximately $14 \mathrm{~mm}$, and the axial depth of the anterior chamber was approximately $6 \mathrm{CT}$. Iris texture was not clear, with partial pigment loss. Extensive iridodialysis was observed, with the formation of a massive iris hiatus. The pupil had upward displacement, with ectropion uveae of the pupil margin and slight lens opacity (Fig. 2 A, B). The clinical features of the pedigree members are shown in Table 1.

\subsection{Sanger sequencing and pathogenicity analysis of mutation sites}

In our study, target capture high-throughput sequencing of 50 known candidate genes for iris disease using capture plates and gDNA samples from the proband was successfully carried out (Fig. 3). A heterozygous missense mutation (c.246C >A) was identified in this proband (nucleotide 246 in the coding region mutated from cytosine to adenine), leading to amino acid changes in p.S82R (amino acid no. 82 changed from serine to arginine), resulting in a missense mutation (Table 2). SIFT, PolyPhen 2, MutationTaster, GERP++ and REVEL were used to predict the pathogenic nature of the c.246C >A (p.S82R) mutation. The results showed that c. $246 \mathrm{C}>\mathrm{A}$ was rated as "deleterious" in SIFT, indicating that amino acid substitution at this conserved site was more likely to affect protein function. In PolyPhen-2, the mutation had a score of greater than 0.95, 
indicating that the mutation was likely to cause changes in protein structure or function. The mutation site was evaluated with Mutation Taster, with the assessment grade of "disease causing". GERP++ had a score greater than 2, indicating that it was conserved, and the more conserved the site was, the greater the effect was on the protein. The REVEL score of a single missense variant ranged from 0 to 1 , and the higher score reflected the higher possibility of disease caused by the variant. The score in REVEL was 0.968, indicating that the mutation might be pathogenic. Based on the above results, the mutation was predicted in the protein function prediction softwares SIFT, PolyPhen 2, MutationTaster, GERP ++ and REVEL as deleterious, deleterious, deleterious, deleterious and deleterious respectively.According to the Standards and Guidelines for the Interpretation of Sequence Variants by the American College of Medical Genetics and Genomics and the Association for Molecular Pathology in 2015, "likely pathogenic" was considered.

3.3 Mutation validation and cosegregation analysis

In our study, mutation identification and cosegregation analysis were completed using deoxy DNA sequencing (Fig. 3). The variant c.246C $>$ A of FOXC1 was identified as a heterozygote in the proband (Table 2). The father and mother of the proband had wild-type genes and normal phenotypes, and the brother and grandmother also had wild-type genes and normal phenotypes. Thus, it was demonstrated that the c.246C $>\mathrm{A}$ variant of the FOXC1 gene co-segregated with the disease phenotype in this pedigree. This mutant was not present in the 100 normal racially matched controls (data not shown). In brief, these findings demonstrated complete segregation of this mutation in this family with Axenfeld-Rieger syndrome and determined its role in the pathogenesis of the disease.

3.4 Conservation analysis of the c. $246 \mathrm{C}>\mathrm{A}$ variant of $\mathrm{FOXC} 1$ and predictive analysis of the $3 \mathrm{D}$ model of the protein

By searching the Conserved Domain Database of the NCBI, gene conservation among different species was analyzed (Fig. 4). This study found that site p.S82 in the FOXC1 gene for serine(S) was highly conserved among species, such as human (NP_001444.2), house mouse (NP_032618.2), rat (NP_599165.1), zebrafish (NP_571803.1), and African clawed frog (NP_001007864.1), suggesting that this amino acid site might play an important role in the function of FOXC1.

SWISS-MODEL was used to construct the three-dimensional structure of the FOXC1 region, and the results showed that the wild-type amino acid at position 82 was serine, which changed to arginine after mutation. The change from noncharged amino acids to positively charged amino acids might affect the function of proteins (Fig. 5).

\section{DISCUSSION}

ARS is a rare genetic heterogeneous disorder characterized by a broad range of ocular and systemic abnormalities (Ito et al.,2014). In our current study, the heterozygous mutation c.246C $>$ A(p.s82r) in the FOXC1 gene was found in the pedigree. ARS is often considered autosomal dominant, and some sporadic cases have also been reported. In this pedigree, the mutation in the FOXC1 gene was identified in the proband, and only the proband had typical signs and symptoms, while other members were normal, which indicated that the variant may be a spontaneous mutation. When inquiring about the family history, we knew that the maternal great grandmother and great grandmother of the proband were sisters, that is, the parents of the proband were consanguineous. Whether this may be the cause of spontaneous mutation of the gene remains to be further studied.

The specific function of the FOXC1 gene has not been fully elucidated. It is known to play an extremely important role in the regulation of embryonic and eye development, and FOXC1 mutations are frequently associated with Axenfeld-Rieger anomaly defined by anterior segment dysgenesis (ASD) with characteristic posterior embryotoxon, iris hypoplasia, and corectopia (Seifi et al.,2018). As an autosomal genetic dysplasia, ARS might manifest as a visual phenotype and/or nonvisual phenotype, and the visual phenotype includes underdeveloped iris stroma, underdeveloped microcornea, anterior synechiae, corneal opacity and juvenile glaucoma. The nonvisual phenotype includes mandibular hypoplasia, dental hypoplasia, abnormal perium- 
bilical skin turnover, hearing impairment, congenital heart disease and renal dysplasia (Reis et al.,2012; Gauthier et al.,2020; Gokce et al.,2015). FOXC1 is also involved in the development of neural crest cells during embryonic development, mutation of which can cause the abnormal structure of aqueous outflow originating from neural crest cells, inducing increased intraocular pressure and blindness (Akula et al.,2019; Seo et al.,2017). In addition, its mutation can be accompanied by systemic abnormalities, such as impairment of facial organs derived from neural crest cells, hearing impairment, and cleft palate. Knockout of the FOXC1 gene in mice caused abnormal development of the eyes, heart, blood vessels, kidney, skeleton and other tissues or organs (Aldinger et al.,2009; Motojima et al.,2017; Motojima et al.,2017b). FOXC1-associated ARS is commonly reported without other systemic abnormalities (Zeynep et al.,2009; Strungaru et al.,2007). However, there are still patients with FOXC1 mutations demonstrating symptoms of systemic dysplasia, including congenital heart defects (CHDs), such as tetralogy of Fallot (Vande et al.,2018), sensorineural hearing loss (Souzeau et al.,2017) and brain abnormalities (Chrystal et al.,2019). The proband in the pedigree involved in our study presented with double eyeball enlargement at birth, was diagnosed with glaucoma, and underwent ventricular septal defect repair in our hospital when he was 3 years old, as well as sublingual cystectomy when he was 5 . In addition, the child had a small tooth deformity and slight skin valgus around the umbilicus, indicating that the child had congenital ventricular septal defects and sublingual cysts. That is, this child had both visual and nonvisual phenotypes.

FOXC1 is expressed in the heart, kidney, eyes and brain; it is a member of the forkhead transcription factor family and plays an important role in embryogenesis, cell migration, cell differentiation, the expression of tissue-specific genes and tumorigenesis (Golson et al.,2016). FOXC1 expresses in heart, kidney, eyes and brain, it was a member of the forkhead transcription factor family and plays an important role in embryogenesis, cell migration, cell differentiation, the expression of tissue-specific genes and tumorigenesis. The forkhead (FH)structural domain of FOXC1 is very conserved and consists of 110 amino acids that recognize and bind to specific DNA sequences to activate target genes. Transcription and activation of downstream genes by the FOXC1 gene requires the involvement of two activation domains of AD-1 and AD-2, and inhibition of domain (ID) can attenuate the activity of these two domains (Lehmann et al.,2003). Mutations in the FOXC1 gene leading to ARS include missense mutations, nonsense mutations and insertion deletions and duplications of small fragments, most of these mutations are in the forkhead domain. There were few reports that the rearrangement of the chromosomal regions where the FOXC1 gene was located caused ARS (D'Haene et al.,2011; Chanda et al.,2008 ) and only one case of $t(6,13)$ equilibrium translocation causing ARS was reported, in which FOXC1 was identified as the pathogenic gene(Nishimura et al.,1998) Patients with chromosome $6 \mathrm{p} 25$ deletion often show eye, cranial, facial, skeletal, heart and kidney deformities, hearing loss, or brain edema(D'Haene et al.,2011;Le Caignec et al.,2005; Descipio et al.,2005). The clinical symptoms of these patients varied, depending on the size of the missing chromosome fragment and the genes it contained. These patients also showed abnormalities in the anterior segment such as postembryonic ring and iris dysplasia, which was due to the absence of the FOXC1 gene or its regulatory elements(Lehmann et al.,2000; Nishimura et al.,2001; Lehmann et al.,2002).In this study, with the help of target capture high-throughput sequencing, a novel heterozygous missense mutation c.246C $>$ A (nucleotide 246 in the coding region mutated from cytosine to adenine)was successfully identified in the proband of this family, resulting in amino acid changes p.S82R (amino acid 82 mutated from serine to arginine). Pathogenicity prediction results of this missense mutation of the FOXC1 gene c.246C $>$ A (p.s82r) suggested that the mutation was identified as pathogenic. After filtering in the HGMD database, this mutation was identified as novel, thus extending its mutation spectrum.

ARS is often autosomal dominant, and some sporadic cases have been reported. In this ARS family, a new heterozygous mutation in the FOXC1 gene was identified only in the proband, while no FOXC1 gene mutation was identified in other family members, suggesting that the missense mutation c.246C $>$ A might be sporadic, which may provide a strong basis for further study of this gene. By searching the Conserved Domain Database of NCBI, gene conservation among different species was analyzed. In our study, it was found that site p.S82 of the FOXC1 gene for serine $\mathrm{S}$ was highly conserved among species such as humans, house mice, rats, zebrafish, and African clawed frogs, suggesting that changes in this amino acid site might play 
an important role in the function of FOXC1. SWISS-MODEL was used to construct the three-dimensional structure of the FOXC1 region, and the results showed that the wild-type amino acid at position 82 was serine, which changed to arginine after mutation. The change from noncharged amino acids to positively charged amino acids might affect the function of proteins.

In summary, the heterozygous missense mutation c.246C $>$ A in the FOXC1 gene was found in an ARS patient in this study. This site was highly conserved between the amino acid series of different species and located near the key protein domain. This mutation led to changes in the three-dimensional structure of the protein and was pathogenic. The discovery of this novel mutation further confirms the importance of FOXC1 in ARS, expands the mutation spectrum of the FOXC1 gene, and provides a new reference for further study of FOXC1 gene function.

\section{ACKNOWLEDGMENTS}

This study was supported by the Science and Technology Strategic Cooperation Programs of Luzhou Municipal People's Government and Southwest Medical University (2017LZXNYD-J01).

\section{CONFLICT OF INTERESTS}

There are no conflicts of interest declared.

\section{REFERENCES:}

Akula, M., Park, J.W., West-Mays, J.A. (2019). Relationship between neural crest cell specification and rare ocular diseases. J. Neurosci. Res., 97(1), 7-15.

Aldinger, K.A., Lehmann, O.J., Hudgins, L.(2009).FOXC1 is required for normal cerebellar development and is a major contributor to chromosome 6q25.3Dandy-Walker malformation. Nat Genet,41 (9) : 1037-1042.

Berry, F.B., Saleem, R.A.,Walter, M.A.(2007).FOXC1 tanscriptional regulation is mediated by N -and C-terminal activationdomains and contains a phyophorylated transcriptional inhibitory domain. Biol Chem,277( 12) : 10292-10297.

Chrystal, P.W.,Walter, M.A.(2019).Aniridia and Axenfeld-Rieger Syndrome: Clinical presentations, molecular genetics and current/emerging therapies.Exp. Eye Res., 189: 107815.

Chanda, B.,Asai-Coakwell, M.,Ye, M.,Mungall, A.J., Barrow, M., Dobyns, W.B.,...Lehmann, O.J.(2008).A novel mechanistic spectrum underlies glaucoma-associated chromosome $6 \mathrm{p} 25$ copy number variation. Hum Mol Genet,17(22): 3446-3458.

Deml, B., Reis, L.M., Maheshwari, M., Griffis, C., Bick, D., \& Semina, E.V. .(2014). Whole exome analysis identifies dominant $\backslash \backslash \mathrm{r}$, col4a $1 \backslash \backslash \mathrm{r}$, mutations in patients with complex ocular phenotypes involving microphthalmia.Clinical Genetics, 86(5), 475-481.

D'Haene, B.,Meire, F., Claerhout, I., Kroes, H. Y., Plomp, A., Arens , Y.H.,...De Baere , E.(2011).Expanding the Spectrum of FOXC1 and PITX2 Mutations and Copy Number Changes in Patients with Anterior Segment Malformations. Investigative Ophthalmology \& Visual Science 52: 324-333.

Descipio, C.,Schneider, L., Young, T. L., Wasserman, N., Yaeger, D., Lu, F.,.. Krantz, I.D.(2005).Subtelomeric deletions of chromosome 6p: molecular and cytogenetic characterization of three new cases with phenotypic overlap with Ritscher-Schinzel (3C) syndrome. Am J Med Genet A, 134A(1): $3-11$.

Gauthier, A.C.,Wiggs J.L.(2020).Childhood glaucoma genes and phenotypes: Focus on FOXC1 mutations causing anterior segment dysgenesis and hearing loss.Exp. Eye Res., 190: 107893.

Gokce, G. , Oren, N. C. , \& Ozgonul, C.(2015). Axenfeld-rieger syndrome associated with severe maxillofacial and skeletal anomalies. Journal of Oral \& Maxillofacial Pathology, 19(1), 109. 
Golson, M.L., Kaestner, K.H.(2016). Fox transcription factors: From development to disease[J]. Development,143(24):4558-4570.

Ito, Y. A., Walter, M.A.(2014).Genomics and anterior segment dysgenesis: a review.Clinical \& Experimental Ophthalmology, 42(1):13-24.

Lehmann, O.J., Sowden, J.C., Carlsson, P., Jordan, T., Bhattacharya, S.S.(2003). Fox's in development and disease.Trends in Genetics, 19(6), 339-344.

Le, Caignec.C.,De, Mas.P.,Vincent, M.C.,Boceno, M.,,Bourrouillou, G., Rival, J.M., David, A.(2005).Subtelomeric 6p deletion: clinical, FISH, and array CGH characterization of two cases. Am J Med Genet A,132A(2): 175-180.

Lehmann, O.J., Ebenezer, N.D., Jordan, T., Fox, M., Ocaka, L., Payne, A.,...Bhattacharya, S.S.(2000). Chromosomal duplication involving the forkhead transcription factor gene FOXC1 causes iris hypoplasia and glaucoma. Am J Hum Genet, 67(5): 1129-1135.

Lehmann, O. J., Ebenezer,N. D., Ekong,R., Ocaka, L., Mungall, A.J., Fraser, S.,... Jordan, T.(2002).Ocular developmental abnormalities and glaucoma associated with interstitial $6 \mathrm{p} 25$ duplications and deletions. Invest Ophthalmol Vis Sci, 43(6): 1843-1849.

Motojima, M., Kume, T., Matsusaka, T.(2017).Foxc1 and Foxc2 are necessary to maintain glomerular podocytes. Experimental Cell Research,352(2):265-272.

Motojima, M., Tanimoto, S., Ohtsuka, M., Matsusaka,T., Kume, T., Abe, K.(2016). Characterization of Kidney and Skeleton Phenotypes of Mice Double Heterozygous for Foxc1 and Foxc2. Cells Tissues Organs (Print), 201(5), 380-9.

Nishimura, D.Y.,Swiderski, R.E.,Alward, W.L. Searby, C.C., Patil, S.R., Bennet, S.R.,...Sheffield, V.C. (1998). The forkhead transcription factor gene FKHL7 is responsible for glaucoma phenotypes which map to 6p25.Nat Genet, 19(2): 140-147.

Nishimura, D.Y.,Searby, C.C.,Alward, W.L., Walton, D., Craig , J.E., Mackey, D.A.,...Sheffield , V.C.et al.(2001). A spectrum of FOXC1 mutations suggests gene dosage as a mechanism for developmental defects of the anterior chamber of the eye.Am J Hum Genet,68( 2) : 364-372.

Reis, L.M., Tyler, R.C., Volkmann Kloss, B. A. ,Schilter, K.F.,Levin, A.V.,Lowry, R. B., ...Semina, E.V.(2012).Pitx2 and foxc1 spectrum of mutations in ocular syndromes. European Journal of Human Genetics, 20(12), 1224-1233.

Sauer, A.,Speeg-Schatz, C.(2012).[Axenfeld-Rieger syndrome].Journal Francais Dophtalmologie,35(5):392.

Seifi, M.,Walter, M.A.(2018).Axenfeld-Rieger syndrome.Clin. Genet., 93(6): 1123-1130.

Seifi, M.,Walter, M.A.(2018). Accurate prediction of functional, structural, and stability changes in PITX2 mutations using in silico bioinformatics algorithms. PLoS ONE, 13(4), e0195971.

Saleem, R.A., Banerjee-Basu, S., Murphy, T.C., Baxevanis, A., \& Walter, M. A.(2004). Essential structural and functional determinants within the forkhead domain of FOXC1.Nucleic Acids Research,32(14): 4182-93.

Seo, S., Chen, L.S., Liu, W,Z., Zhao, D.M., Schultz, K.M., Sasman, A., ...Kume, T.(2017). Foxc1 and Foxc2 in the Neural Crest Are Required for Ocular Anterior Segment Development. Invest. Ophthalmol. Vis. Sci., 58(3), 1368-1377.

Strungaru, M.H., Dinu, I., Walter, M.A.(2007).Genotype-Phenotype Correlations in Axenfeld-Rieger Malformation and Glaucoma Patients with FOXC1 and PITX2 Mutations. Investigative Ophthalmology \& Visual Science,48(1):228-237. 
Souzeau, E., Siggs, O.M., Zhou, T., Galanopoulos, A., Hodson, T., Taranath, D.,... Craig, J.E.(2017). Glaucoma spectrum and age-related prevalence of individuals with FOXC1 and PITX2 variants. Eur. J. Hum. Genet., 25(11), 1290.

Vande, P.P., Zazo, S.C., Patat, O., Bouneau, L., Vigouroux, A., Bourgeois, D., ...Calvas, P.(2018). 4q25 microdeletion encompassing PITX2: A patient presenting with tetralogy of Fallot and dental anomalies without ocular features. Eur J Med Genet, 61(2), 72-78.

Zeynep, T., Daniella, B.(2009). Axenfeld-rieger syndrome and spectrum of pitx2 and foxc1 mutations.European Journal of Human Genetics Ejhg, 17(12), 1527-1539.

Figure and table captions

Figure1 Pedigree with Axenfeld-Rieger syndrome in the proband. Family members and disease-causing mutations are presented. Normal individuals are shown as clear circles (females) or squares (males), whereas the affected individual is shown as a filled square with an arrow indicating the proband (III:2) with the missense variant of the FOXC1 gene: c.246C > A. "M" indicates the mutant allele of FOXC1 (mutant type), whereas "+" indicates the normal allele (wild type).

Figure 2 Representative anterior segment photography of patient III:2 from both eyes. A and B represent the right eye and left eye, respectively. C shows systemic and ocular characteristics of patient III:2. D shows developmental malformation of the teeth, while E shows the outward projection of the navel.

Figure 3 Pyrogram profiles for mutation verification by dideoxy DNA sequencing shows overlapping peaks at nucleotide position 246 (red arrow) due to the missense variant of the FOXC1 gene: c. $246 \mathrm{C}>\mathrm{A}$. in the proband (III:2), while the variant was not detected in the proband's parents (II:1, II:2), his brother (III:1) or his grandmother (I:2). Unfortunately, DNA samples of his grandfather (I: 1) were not collected.

Figure 4 Multiple sequence alignment of the region of the FOXC1 protein surrounding the novel p.S82R mutation in various species. The serine(s) residue (indicated with a red strip-type frame) was highly conserved among all species analyzed.

Figure 5 The results showed that the 82 nd amino acid of the wild type was serine, which changed to arginine after mutation, leading to an uncharged amino acid (A, wild type) change to a positively charged amino acid (B, p.S82R).

Table 1 Clinical features of the pedigree members. Abbreviations: VSD, ventricular septal defect

Table 2 Characteristics of the FOXC1 variant in the proband and analysis of its disease-causing effects. Abbreviations: c, variation at the cDNA level; p, variation at the protein level; S82R, serine substitution conserved arginine at codon 82; hetero, heterozygote; AD, autosomal dominant inheritance.

Table 3 PCR primers and PCR product size 


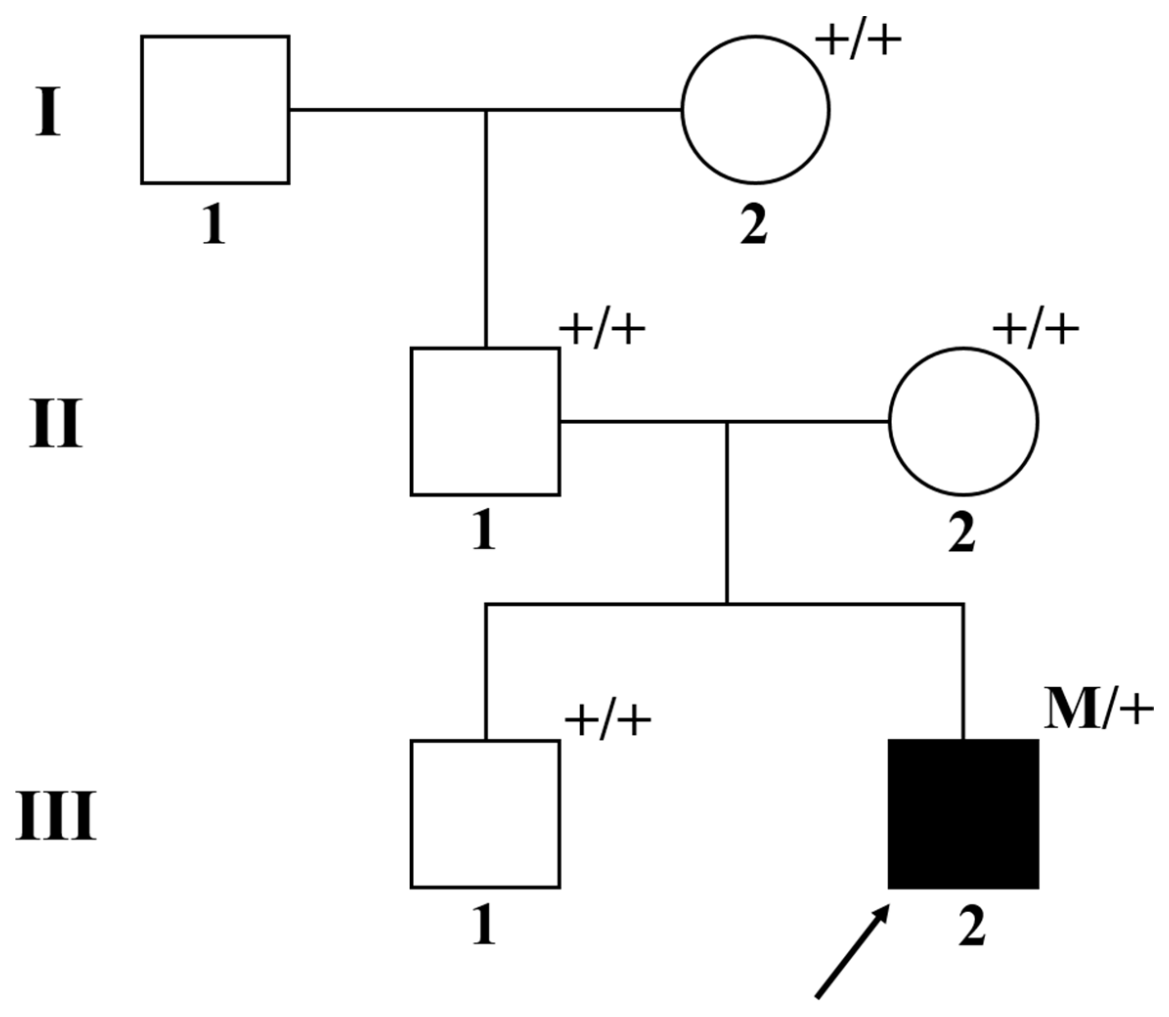

FOXC1: c.246C $>$ A

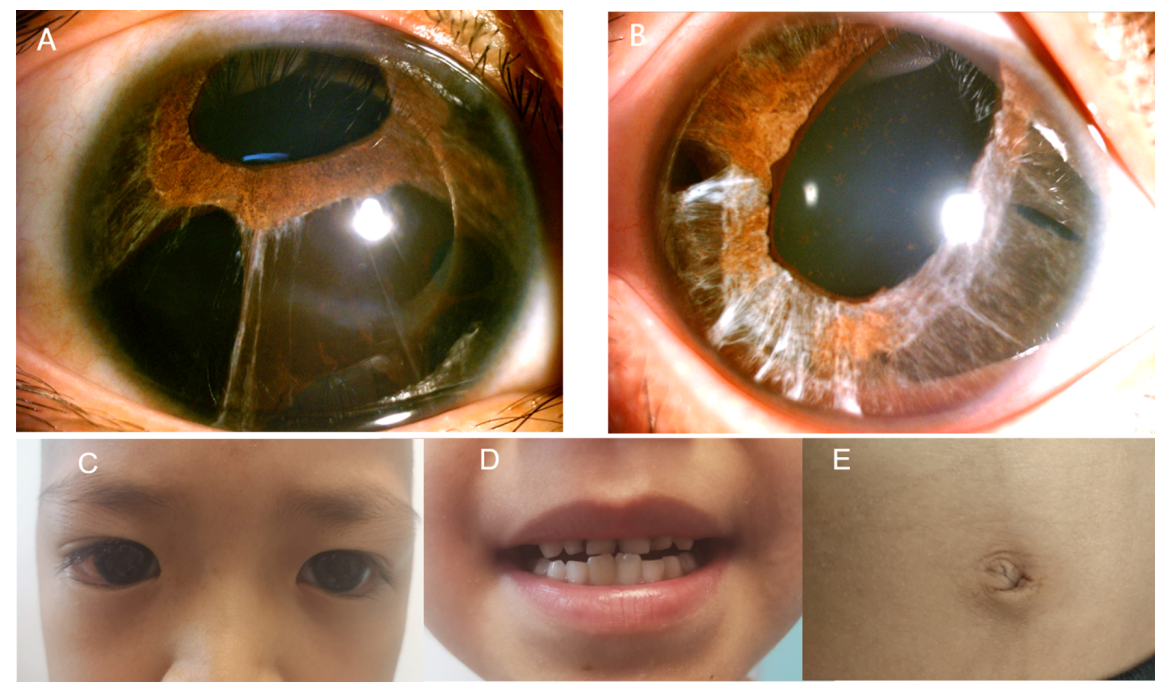



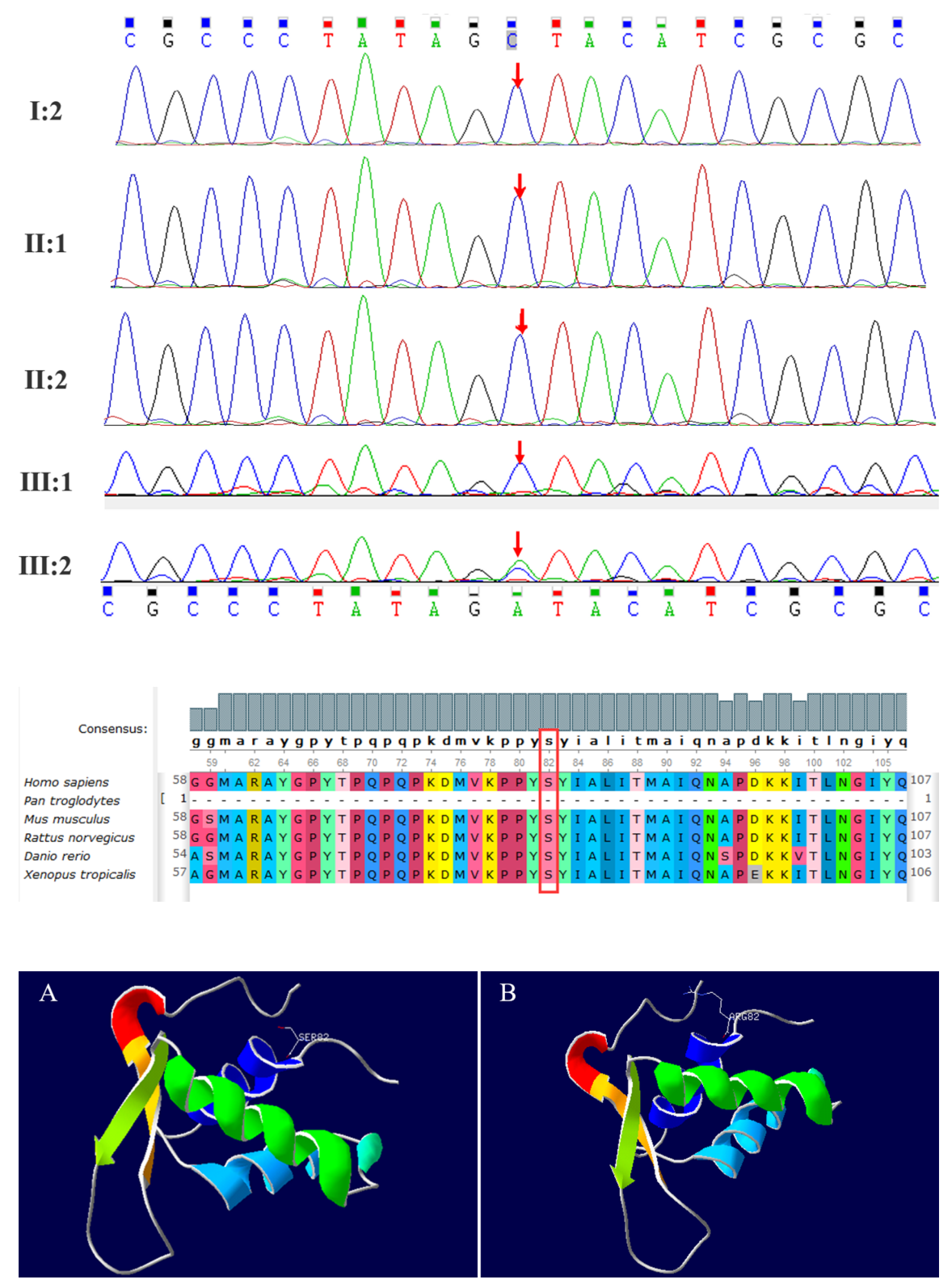

\section{Hosted file}

Table 1.docx available at https://authorea.com/users/329227/articles/456272-a-novel-variantof-the-foxc1-gene-causes-axenfeld-rieger-syndrome-with-congenital-glaucoma-in-a-chineseboy

\section{Hosted file}

Table 2.docx available at https://authorea.com/users/329227/articles/456272-a-novel-variantof-the-foxc1-gene-causes-axenfeld-rieger-syndrome-with-congenital-glaucoma-in-a-chineseboy

\section{Hosted file}


Table 3.docx available at https://authorea.com/users/329227/articles/456272-a-novel-variantof-the-foxc1-gene-causes-axenfeld-rieger-syndrome-with-congenital-glaucoma-in-a-chineseboy 